LOGISTIEK \& Tijdschrift voor toegepaste logistiek 2019 nr. 7

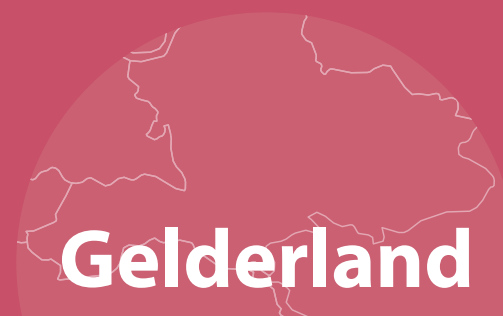

Nursing homes face the challenge to develop task assignment strategies that enable them to timely and efficiently meet the demand of their clients. 


\title{
Demand-Driven Task- Scheduling in a Nursing Home Setting: A Genetic Algorithm Approach
}

René Bekker

Dennis Moeke

Nanne Dieleman, Martijn Buitink, Jarik den Uijl, Floor Otsen, Kilian Koreman, Reven Passial

Maik Couwenberg
Assistant Professor at VU University Amsterdam,

Department of Mathematics

Acting Professor at HAN University of Applied Sciences

Student Business Analytics, VU University Amsterdam, Department of Mathematics

\begin{abstract}
Nursing homes face the challenge to develop task assignment strategies that enable them to timely and efficiently meet the demand of their clients. In this contribution, we analyze a practice-based task scheduling problem with small time windows and care workers with different qualification levels. A set of care tasks has to be assigned to a given number of care workers with the objective of delivering the care as close as possible to the time preferences of the nursing home clients. To solve this problem, we propose a scheduling approach in which a Genetic Algorithm is combined with an Integer Linear Program. We verified the approach using numerical experiments. The next step will be to test the algorithm with reallife data and to implement it in practice.
\end{abstract}




\section{Introduction}

As in many Western countries, the population in the Netherlands is ageing. The percentage of people aged 65+ is expected to rise from 19\% in 2018 to $29 \%$ in 2040. In addition, currently around $5 \%$ of the total population is aged 80 years or over. By 2040 , this share is expected to reach $10 \%$ [1][2].

This change in demographics puts pressure on the financial sustainability of the Dutch long-term care (LTC) system because of two main reasons. Firstly, the need of care increases with age. For example, on average, people over the age of 85 consume four times as much healthcare per person as those aged between 65 and 75 (NZa, 2018). Recent projection scenarios show a non-negligible increase in public spending on LTC over the forthcoming decades (RIVM, 2018). Secondly, it will lead to a substantial increase of the old-age dependency ratio, which measures the number of elderly people at an age when they are generally economically inactive, as share of those of working age. In order to ensure the long-term financial sustainability of the Dutch LTC system, providers of long-term care are challenged to provide their care services in a more efficient manner.

As in most European countries, long-term care is provided both in institutions (residential care) and in communities (home care). In this study, the primary focus is on institutional care provided in nursing homes. A nursing home can be described as a facility with a domestic styled environment that provides 24-hour functional support and care for persons who require assistance with activities of daily living and who often have complex health needs and increased vulnerability (Sanford et al., 2015, p.183). It should be noted that in this contribution no distinction is made between nursing homes and residential homes (less intensive care) as, in practice, the boundary between the two is diffuse (Hamers, 2011).

Often, nursing home clients are in need of ongoing assistance with basic activities of daily living due to physical or psychological disabilities. Consequently, in order to live their lives according to their own daily routines, nursing home clients depend greatly on whether their time preferences regarding care and support services are being met. As such, quality of care in a nursing home largely depends on the coordination and timing of service delivery. In practice, when it comes to the coordination and timing of service delivery, nursing homes have to balance the goal of meeting clients' preferences with the efficient use of resources. In this context, care workers are by far the most important resource, see e.g., Bekker, Moeke \& Schmidt, 2018.

A prominent question that arises in this context is: which of the available care workers should perform which care tasks at which time of the day in order to meet the demand of the nursing home clients as closely as possible? In current nursing home practice, decisions regarding the allocation of care workers are often addressed without a sound quantitative basis (e.g., Bekker, Moeke \& Schmidt, 2018; Moeke, Bekker \& Koole, 2017). In this contribution 
we mainly focus on care by appointment. These are care tasks for which, based on the needs and preferences of the client, it is possible to make a fairly detailed planning in advance (Moeke, 2016). Examples are giving medicine and help with getting out of bed in the morning (care by appointment). Hence, we here neglect care on demand consisting of care tasks that cannot be scheduled beforehand and is inherently of a random nature, see e.g. Van Eeden et al., 2014.

In this contribution we elaborate on a practice-based nursing home task scheduling problem with small time windows and care workers with different qualification levels (QLs). We propose a Genetic Algorithm (GA) for determining which care worker (and of which QL) should perform which task in which order. The shifts for the care workers are assumed to be known. The optimal starting times for the care tasks are determined by an Integer Linear Program (ILP) that is incorporated in the GA. The objective of the described task-scheduling method is to assign care tasks to the available and qualified care workers as close as possible to the preferred start times of the nursing home clients.

The overall purpose of this study was to develop a task-scheduling method which has to potential to be implemented in practice.

The remainder of this paper is structured as follows. In the following section, we provide an overview of related literature. In Sections 3 we elaborate on the underlying optimization problem and present a GA approach to tackle the described problem. Next, in Section 4 we verify the model. Section 5 concludes this paper and points out possible directions for further research.

\section{Related literature}

Task scheduling is often considered to be a subproblem of workforce planning, see e.g., Ernst et al., 2004. According to Ernst et al. (2004, p. 5), "Tasks are usually defined in terms of a starting time and duration, or a time window within which the task must be completed, and the skills required to perform the task". Furthermore, they state that task scheduling can be described as combining "individual tasks into task sequences that could usefully be carried out by one person".

The scheduling of tasks by care workers can be interpreted as an unrelated parallel machine scheduling problem, for which there is a vast amount of literature available, see e.g. Allahverdi et al., 2008. Typically, machine scheduling focuses on minimizing the makespan, whereas in this contribution we focus on the delay with respect to preferred starting times. Some prominent examples of task scheduling problems involving time windows are Gertsbakh and Stern (1978) and Mankowska et al. (2014). Gertsbakh and Stern (1978) discuss task scheduling with time windows for a homogeneous workforce. The difference is 
that they do not incorporate delay penalties. Their objective is to find the minimum required staffing requirements to obtain a feasible schedule. Mankowska et al. (2014) consider the more general home care setting where travel times are taken into account. They assume time windows to reflect preferences of the clients. The time windows are half open, as they do not allow for earliness and penalize lateness. The objective is to minimize the weighted sum of total travel times, total tardiness, and maximum tardiness. Their solution is based on an ILP for small instances and a heuristic for larger ones.

Another distinguishing feature of task scheduling in nursing homes is that different qualifications (also referred to as skills) should be taken into account, see for example Bellenguez-Morineau \& N'eron (2007), Krishnamoorthy et al. (2012), Schimmelpfeng et al. (2012). However, Bellenguez-Morineau \& N'eron (2007) focuses on the makespan as objective, whereas Krishnamoorthy et al. (2012) considers the minimum workforce for a feasible schedule in case of hard constraints for start times. Finally, Schimmelpfeng et al. (2012) present a task scheduling approach for rehabilitation hospitals with different qualifications and precedence constraints between tasks, but they do not consider time preferences for individual tasks.

The problem studied in Lieder et al. (2015) is most closely related to the problem at hand. They also focus on the assignment of tasks, with different levels of qualification, in a nursing home setting. To optimally solve this scheduling problem, they propose a mixed integer program (MIP) and a dynamic programming (DP) approach. However, the state space of the presented DP approach has a high dimensionality. As such, the computation time of this approach may become prohibitively long.

\section{Problem and model description}

The task scheduling problem at hand can be described as follows. A given number of care tasks, with predefined durations, have to be assigned to the available care workers. The availability of the care workers is defined in a shift schedule, which is assumed to be known. Each care task has a preferred start time based on the time preference of the nursing home client in question.

Furthermore, each care worker has a qualification level. Based on their education and expertise, care workers are hierarchically divided into three distinct qualification levels (QLs). Depending on the required education and complexity of care, healthcare tasks are assigned to a healthcare worker with that specific qualification level. Qualification level one (QL1) is required to perform the least complex tasks whereas QL3 is required to perform the most complex tasks (see Table 1). 
Table 1 Overview Qualification Levels (QLs) and typical tasks

\begin{tabular}{|c|c|}
\hline Qualification Level (QL) & Typical tasks \\
\hline QL 1 & Bringing food or drinks, cleaning, transferring, bed cleaning \\
\hline QL 2 & $\begin{array}{l}\text { Proving help with: getting out of bed, eating and drinking, toileting, } \\
\text { washing or showering }\end{array}$ \\
\hline QL 3 & Giving medication, simple medical check ups \\
\hline
\end{tabular}

To obtain a feasible task schedule, it has to be ensured that each task is assigned to a care worker with (at least) the required qualification level; the start time is preferably as close as possible to the preferred start time. A care worker can only perform one task at a time and preemption of tasks is not allowed. The objective is to create a feasible task schedule that minimizes a weighted combination of the total earliness and waiting time for nursing home clients.

In order to solve the described task scheduling problem, we iteratively carry out a two-step procedure. In each iteration, we first determine which care worker should perform which task in which order. In this step we look for new candidate solutions using a Genetic Algorithm (GA). Then, we determine the optimal starting times for the care tasks. For this step an ILPapproach (for each care worker) is used. These two steps together yield the fitness of a candidate solution and are used in the GA to find better solutions.

In this section we explain the approach using the example as described in the textbox bellow. The first elementary step is the representation of a task schedule, such that it is amenable for a GA, after which the genetic algorithm can be carried out. 


\section{Example}

On a particular morning, three care workers are available in nursing home department $A$. They have to complete six activities for five clients. The details are provided in Tables 2 and 3.

Table 2 Shift schedule care workers

\begin{tabular}{|c|c|c|c|c|}
\hline ID & Name & $\begin{array}{l}\text { Qualification } \\
\text { Level (QL) }\end{array}$ & Start time & End time \\
\hline 1 & Mike & 3 & 7:00 & $8: 30$ \\
\hline 2 & Clara & 2 & 7:00 & 9:00 \\
\hline 3 & Jacqueline & 3 & 7:00 & $10: 00$ \\
\hline
\end{tabular}

Table 3 Care tasks which have to be performed

\begin{tabular}{|c|c|c|c|c|c|}
\hline ID & $\begin{array}{l}\text { Preferred } \\
\text { Start time }\end{array}$ & Duration & Client & $\begin{array}{l}\text { Care } \\
\text { task }\end{array}$ & $\begin{array}{l}\text { Required } \\
\text { QL }\end{array}$ \\
\hline 1 & 7:15 & 50 & 1 & Showering & 2 \\
\hline 2 & $7: 15$ & 5 & 2 & Medicine & 3 \\
\hline 3 & $7: 30$ & 25 & 2 & Breakfast & 1 \\
\hline 4 & 8:00 & 15 & 3 & Coffee & 1 \\
\hline 5 & $8: 10$ & 50 & 4 & Showering & 2 \\
\hline 6 & 9:00 & 10 & 5 & Dressing & 1 \\
\hline
\end{tabular}

\section{Representation}

A task schedule, also referred to as 'chromosome' in GA terminology, can be described by a matrix representation which is based on Mankowska et al. (2014). An example of a chromosome for department $A$ is presented in Figure 1. Each row represents a care worker and each column a care task. Hence, a chromosome is an $\mathrm{N} \times \mathrm{M}$ matrix, where $\mathrm{N}$ is the number of available care workers and $M$ the number of available care tasks. Each column only contains one task ID, since a task is performed by only one care worker. The chromosome indicates in which sequence the care worker has to perform the assigned care tasks. Thus, employee 2 will first perform task 1, then task 4, and finally task 5 . Note that this does not necessarily mean that task 3 is performed after task 1 is completed. Also, notice that column i contains task i. Each task receives its ID once the tasks have been sorted by preference time (early-late) and by duration (short-long). This ordering was adopted since, for a single care worker with fixed tasks, this evidently is the optimal order in terms of minimal waiting time and earliness. 


\begin{tabular}{|c|c|c|c|c|c|c|c|}
\hline \multirow{5}{*}{$\begin{array}{l}\text { Care } \\
\text { worker }\end{array}$} & \multicolumn{7}{|c|}{ Task } \\
\hline & & 1 & 2 & 3 & 4 & 5 & 6 \\
\hline & 1 & - & - & 3 & - & - & 6 \\
\hline & 2 & 1 & - & - & 4 & 5 & - \\
\hline & 3 & - & 2 & - & - & - & - \\
\hline
\end{tabular}

Figure 1 Example of chromosome for department $A$

\section{Outline GA procedure}

A population is a collection of task schedules, or chromosomes. In each iteration of the GA, a collection of children is created by using 'crossover' and 'mutation' operations on the parents. Subsequently, this new generation of chromosomes consists of better solutions than the old generation, which explains the evolutionary character of the algorithm. The following pseudo code, based on Eiben \& Smith (2003), describes the general scheme of the genetic algorithm; steps 2 - 5 are further explained below:

BEGIN

INITIALISE population with random candidate solutions;

REPEAT UNTIL (Time limit is exceeded or solution does not improve for $x$ iterations)

DO

1 SELECT parents at random

2 CROSSOVER pairs of parents

3 MUTATE the resulting offspring

4 EVALUATE new candidates

5 SELECT individuals for the next generation

OD

END 


\section{Crossovers}

A crossover is one of the operations that is performed in order to create new chromosomes. It is performed on two parent chromosomes and creates two children. Figure 2 provides an example of the crossover operation. The operation procedure for two randomly selected parents is as follows:

1. Randomly generate the crossover point $P$.

2. Create child 1 by selecting columns $[1: P]$ of the mother and $[P+1: M]$ of the father.

3. Create child 2 by selecting columns $[1: P]$ of the father and $[P+1: M]$ of the mother.

Mother

\begin{tabular}{l|c|c|c|c|c|c|c|}
\multicolumn{1}{c}{ Task } \\
\cline { 2 - 9 } $\begin{array}{l}\text { Care } \\
\text { worker }\end{array}$ & 1 & - & - & 3 & - & - & 6 \\
\cline { 2 - 9 } & 2 & 1 & - & - & 4 & 5 & - \\
\hline 3 & - & 2 & - & - & - & - \\
\hline
\end{tabular}

Father

\begin{tabular}{l|c|c|c|c|c|c|c|}
\multicolumn{1}{c}{ Task } \\
\cline { 2 - 9 } $\begin{array}{l}\text { Care } \\
\text { worker }\end{array}$ & 1 & 2 & 3 & 4 & 5 & 6 \\
\cline { 2 - 9 } & 1 & - & 2 & - & - & 5 & - \\
\cline { 2 - 9 } & 2 & 1 & - & 3 & - & - & - \\
\hline 3 & - & - & - & 4 & - & 6 \\
\hline
\end{tabular}

Child 1

\begin{tabular}{l|c|c|c|c|c|c|c|}
\multicolumn{1}{c}{ Task } \\
\cline { 2 - 9 } $\begin{array}{l}\text { Care } \\
\text { worker }\end{array}$ & 1 & - & - & 3 & - & 5 & - \\
\cline { 2 - 8 } & 2 & 1 & - & - & 4 & - & - \\
\hline 3 & - & 2 & - & - & - & 6 \\
\hline & &
\end{tabular}

Child 2

\begin{tabular}{l|c|c|c|c|c|c|c|}
\multicolumn{1}{c|}{ Task } \\
\cline { 2 - 9 } $\begin{array}{l}\text { Care } \\
\text { worker }\end{array}$ & 1 & 1 & 2 & 3 & 4 & 5 & 6 \\
\cline { 2 - 8 } & 2 & 1 & - & 3 & - & 5 & - \\
\cline { 2 - 8 } & 3 & - & - & - & 4 & - & - \\
\hline
\end{tabular}

Figure 2. Crossover example for department $A$. The red line represents the crossover point $P$.

\section{Mutations}

The second operations that are performed to create new chromosomes are mutations. With a certain probability, a child is mutated according to the following procedure:

1. Randomly select a column (i.e., a task) to mutate.

2. Randomly select a row (i.e., a care worker) with the correct qualification level.

3. Assign the task to this new care worker.

4. Repeat this procedure $X$ times 
The number of mutations performed on each chromosome differs each time. Tests indicate that it works well is if $X$ is sampled from the range $\{1, \ldots, 5\}$.

An example of the mutation operation is illustrated in Figure 3. In this example, task 2 is selected which is currently assigned to care worker 3 . Subsequently, row 1 (care worker 1 ) is selected and task 2 is moved to this row. In the new schedule, no tasks are assigned to care worker 3, whereas three tasks are assigned to care worker 1.

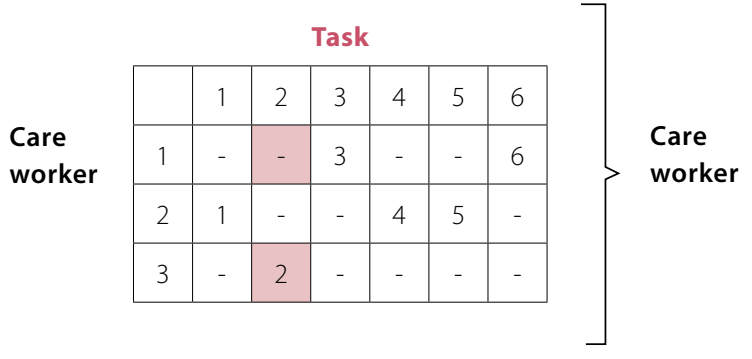

Task
\begin{tabular}{|c|c|c|c|c|c|c|}
\hline & 1 & 2 & 3 & 4 & 5 & 6 \\
\hline 1 & - & 2 & 3 & - & - & 6 \\
\hline 2 & 1 & - & - & 4 & 5 & - \\
\hline 3 & - & - & - & - & - & - \\
\hline
\end{tabular}

Figure 3 An example of a mutation operation for department A.

\section{Evaluation, fitness function and ILP}

The fitness function assigns a value to the chromosome that indicates how good the solution is. The fitness consists of three elements:

4. The sum of the total earliness and waiting time of all tasks in the schedule (determined by the solution of an ILP per care worker below).

5. A penalty when the solution is infeasible.

6. A penalty when the last task of a care worker plus the duration of the task exceeds the end time of the shift of the concerning care worker.

The penalties have considerable large values to make sure that infeasible schedules are excluded from the consecutive generations. As the chromosomes only provide which care worker carries out which task and in which order, a crucial element is to determine the best starting times of tasks. This is accomplished using an ILP for each care worker, which we discuss next.

Consider a care worker that has to carry out $n$ tasks. These $n$ tasks have the following characteristics:

$G_{i}=$ preferred start time of care task (in minutes), $i=1, \ldots, n$

$D_{i}=$ expected duration of care task (in minutes), $i=1, \ldots, n$ 
The objective is to minimize the weighted sum of earliness and waiting time. Let $C_{W}$ and $C_{E}$ denote the coefficients for waiting and earliness, which may vary between 0 and 1 and have the relation that $C_{W}=1-C_{E}$. If both values are equal to 0.5 , the clients have no preference regarding waiting or being helped early. If $C_{W}$, for example, is equal to $0.3, C_{E}$ is equal to 0.7 and the clients prefers waiting over earliness.

To determine optimal starting times, we define the following decision variables:

$x_{i}=$ actual start time of the care task (in minutes), $i=1, \ldots, n$

Observe that the waiting time for the $i$-th task equals $\max \left(0, x_{i}-G_{i}\right), i=1, \ldots, n$. To incorporate this in a linear model, we use the auxiliary variables $y_{i}, i=1, \ldots, n$. Similarly, the earliness of the $i$-th task is $\max \left(0, G_{i}-x_{i}\right), i=1, \ldots, n$, which is incorporated in our ILP using the auxiliary variables $y_{i}, i=n+1, \ldots, 2 n$. This give rise to the following ILP for each care worker

$$
\text { Minimize } C_{W} \sum_{i=1}^{n} y_{i}+C_{E} \sum_{i=n+i}^{2 n} y_{i}
$$

subject to:

$x_{i} \geq$ Begin_Shift

$x_{i}+D_{i} \leq x_{i}+{ }_{1}, i=1, \ldots, n$

$y_{i}-x_{i} \geq-G_{i}, i=1, \ldots, n$

$y_{i}+x_{i} \geq-G_{i} i=n+1, \ldots ., 2 n$

$x_{n} \leq$ End_Shift

$x_{i} \geq 0, i=1, \ldots ., n$

$y_{i} \geq 0, i=1, \ldots ., 2 n$

\section{Select}

Once all children have been generated and the corresponding fitness values are computed, a new generation with the same size as the current generation is created. It consists of $95 \%$ of the children and parents that have the highest fitness values. $5 \%$ of the new generation is obtained from solutions that have a higher fitness value. This is done to reduce the probability that the GA ends up in a local minimum. Chromosomes that do not make it to the new generation are deleted. Once the new generation is created, the process of creating new children by crossover and mutation operations is repeated. 


\section{Extension: breaks care workers}

In the proposed model, we assume that a care worker has one break per shift. Regarding the timing of the break, a care worker can indicate his or her time preference and the duration. The break is scheduled as close as possible to the preferred time. However, when there are tasks with the same preferred time as the break, these tasks receive priority. If a break starts earlier or later than the preferred time, no waiting time or early time is incurred, since the break times are flexible and the employee only indicates a preference. The breaks are added to the list with care tasks that need to be scheduled. The GA then also schedules the employee breaks. In order to make sure that the employee breaks do not receive any priority over the care tasks, the ILP coefficients of the variables belonging to the breaks are 0 .

\section{Verification}

The algorithm, as described in Section 3, was developed in R (version 3.5.1). During the development of the algorithm, all elements of the algorithm have been tested by using test cases with a known solution. For the final validation, over 30 test cases were created and run successfully.

We now show the result of two test cases. First, we present the outcome of the algorithm for department A that we introduced in Section 3 (see Figure 4). For this example, we used the data presented in Tables 2 and 3 as input. It can be observed that all 6 tasks are assigned to a qualified care worker according to the preferred starting time of clients. Hence, no client is served to early or late. Figure 5 shows the result of a more complex test case. The input files of this test case can be found in Appendix 1 (Tables 4 and 5). The algorithm was able to find an optimal solution in 15.1 minutes (on an Intel i5 CPU with $2.6 \mathrm{GHz}$ and $8 \mathrm{~GB}$ RAM) with a total earliness and waiting time of 0 minutes. 


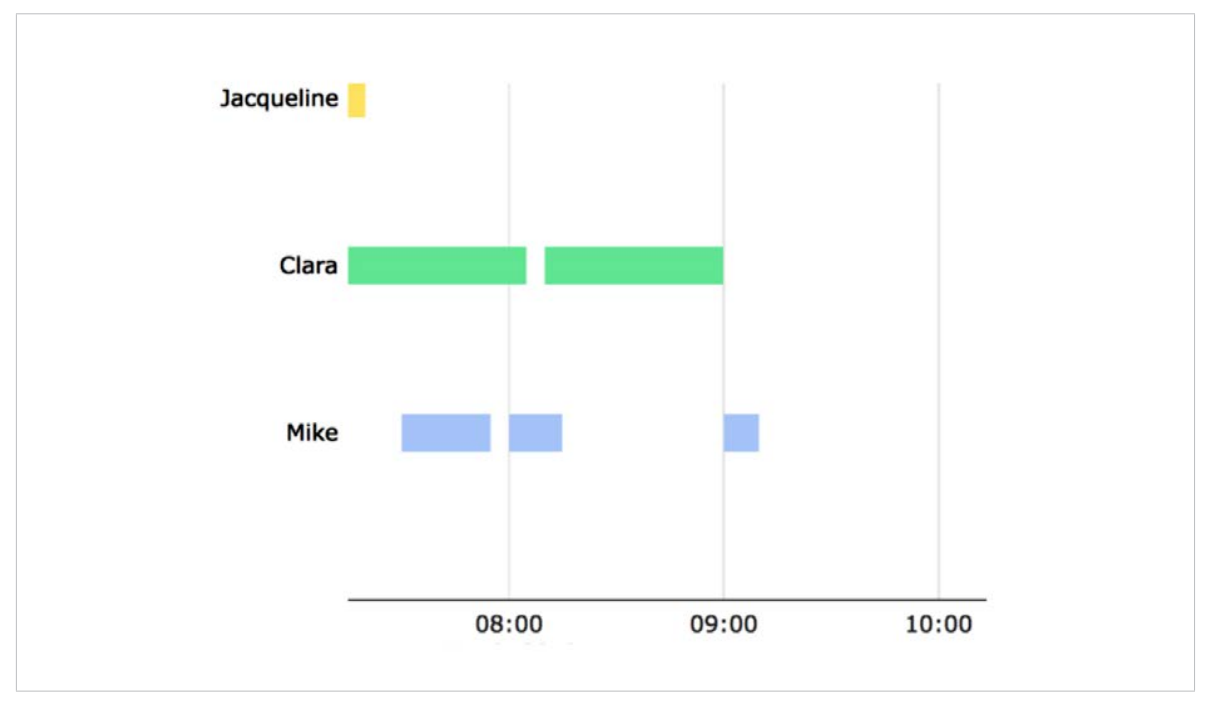

Figure 4 Example of task schedule for department $A$

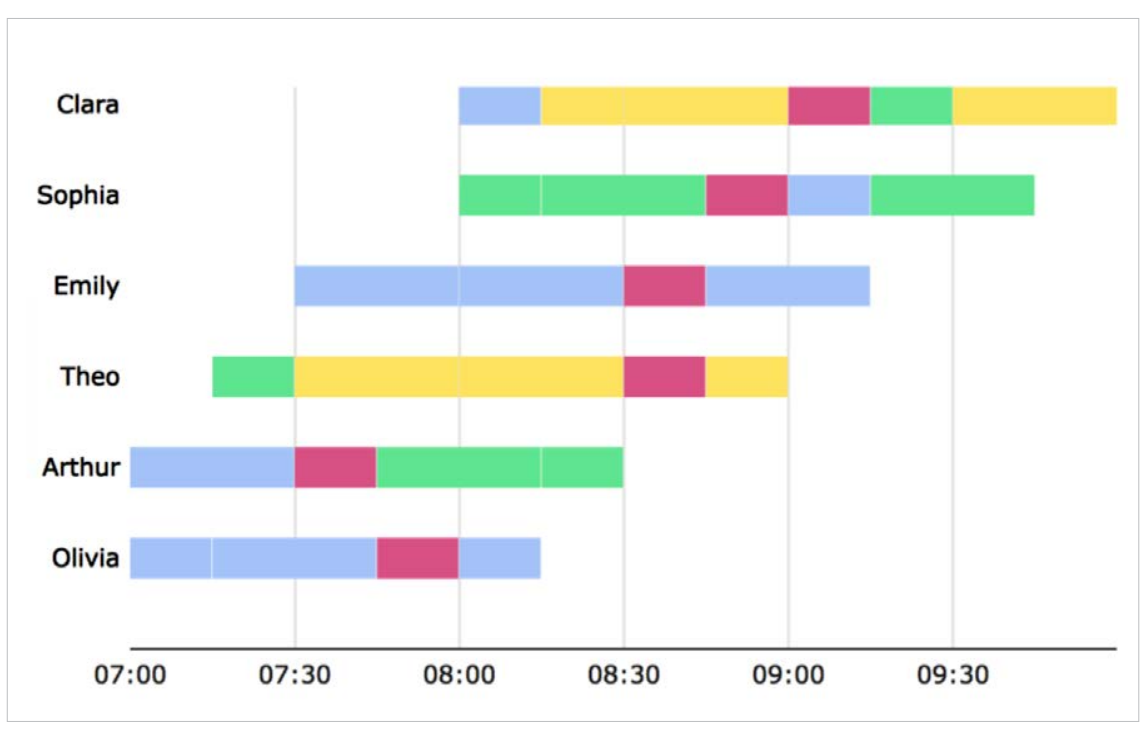

Figure 5 Optimal schedule test case Appendix 1. Red horizontal bars indicate breaks. 


\section{Conclusions and further research}

In this contribution we elaborate on a practice-based nursing home task scheduling problem with small time windows and care workers with different qualification levels (QLs). We propose a scheduling approach in which a Genetic Algorithm is combined with an ILP in order to obtain schedules in which tasks are assigned to the available care workers as close as possible to the preferred start times of the nursing home clients (i.e., minimizing the total earliness and waiting time). The verification results show that our approach provides feasible (often optimal) schedules in short computation times.

In daily nursing home practice, staff allocation decisions are often made without using mathematically-based methods. The scheduling approach proposed in this contribution could be helpful in assigning care tasks in a more efficient manner. It should be noted, that the shift schedule is used as input and assumed to be available. In practice, this is not always the case. As such, combining the presented approach with shift scheduling could be a fruitful direction for further research. Furthermore, it could also be valuable to extend the model by including random care tasks (i.e., care on demand) and walking distances.

The first next step would be to perform a more extensive numerical study on larger problem instances using real-life data. These data are currently being collected. After this more extensive numerical study, an empirical study will be conducted in which the developed taskscheduling approach will be tested in practice. In this empirical study, both the technical- as well as the human and organizational aspects will be considered.

\section{Literature}

[1] https://www.statista.com/statistics/519754/population-of-the-netherlands-by-age/

[2] https://www.populationpyramid.net/netherlands/2050/

Allahverdi, A., Ng, C. T., Cheng, T. E., \& Kovalyov, M. Y. (2008). A survey of scheduling problems with setup times or costs. European journal of operational research, 187(3), 985-1032.

Bekker, R., Moeke, D., \& Schmidt, B. (2018). Keeping pace with the ebbs and flows in daily nursing home operations. Health care management science, 1-14.

Bellenguez-Morineau, O., \& Néron, E. (2007). A branch-and-bound method for solving multi-skill project scheduling problem. RAIRO-operations Research, 41(2), 155-170.

Eiben, A. E., \& Smith, J. E. (2003). Introduction to evolutionary computing (Vol. 53). Berlin: springer.

Ernst, A. T., Jiang, H., Krishnamoorthy, M., \& Sier, D. (2004). Staff scheduling and rostering: A review of applications, methods and models. European journal of operational research, 153(1), 3-27. 
Gertsbakh, I., \& Stern, H. I. (1978). Minimal resources for fixed and variable job schedules. Operations Research, 26(1), 68-85.

Hamers, J. P. H. (2011). De intramurale ouderenzorg: nieuwe leiders, nieuwe kennis, nieuwe kansen. Den Haag: Raad voor de Volksgezondheid en Zorg.

Krishnamoorthy, M., Ernst, A. T., \& Baatar, D. (2012). Algorithms for large scale shift minimisation personnel task scheduling problems. European Journal of Operational Research, 219(1), 34-48.

Lieder, A., Moeke, D., Koole, G., \& Stolletz, R. (2015). Task scheduling in long-term care facilities: A client-centered approach. Operations Research for Health Care, 6, 11-17.

Moeke, D., Bekker, R., \& Koole, G. (2017) Op tijd naar het toilet door data-gedreven capaciteitsplanning. STAtOR, 4, 8-11.

Moeke, D. (2016), Towards High-Value(d) Nursing Home Care: Providing Client-Centred care in a More Efficient Manner (doctoral dissertation), VU University, Amsterdam. Retrieved from: https://1drv.ms/b/s!AuuRA65uxCQphbl_ZT_GdYOq2VR6iA

Mankowska, D. S., Meisel, F., \& Bierwirth, C. (2014). The home health care routing and scheduling problem with interdependent services. Health care management science, $17(1), 15-30$.

NZa (2018), Zorg voor ouderen 2018, Monitor Nederlandse Zorgautoriteit, Retrieved from: www.rijksoverheid.nl/binaries/rijksoverheid/documenten/rapporten/2018/04/19/ monitor-zorg-voor-ouderen-2018/monitor-zorg-voor-ouderen-2018.pdf

Sanford, A. M., Orrell, M., Tolson, D., Abbatecola, A. M., Arai, H., Bauer, J. M., ... \& Hajjar, R. (2015). An international definition for "nursing home". Journal of the American Medical Directors Association, 16(3), 181-184.

Schimmelpfeng, K., Helber, S., \& Kasper, S. (2012). Decision support for rehabilitation hospital scheduling. OR spectrum, 34(2), 461-489.

Van Eeden, K., Moeke, D., \& Bekker, R. (2016). Care on demand in nursing homes: a queueing theoretic approach. Health care management science, 19(3), 227-240.

RIVM (2018), Volksgezondheid Toekomst Verkennis (VTV) 2018, van https://www.vtv2018.nl/ trendscenario 


\section{Appendix 1}

Table 4 Input 1 file testcase 3

\begin{tabular}{|c|c|c|c|c|}
\hline $\begin{array}{l}\text { Task } \\
\#\end{array}$ & Preferred time & $\begin{array}{l}\text { Estimated dura- } \\
\text { tion (minutes) }\end{array}$ & Client ID & $\begin{array}{l}\text { Required Qualifi- } \\
\text { cation Level }\end{array}$ \\
\hline 1 & $7: 00$ & 15 & 63 & 1 \\
\hline 2 & $7: 15$ & 30 & 45 & 1 \\
\hline 3 & $8: 00$ & 15 & 46 & 1 \\
\hline 4 & $7: 00$ & 30 & 52 & 1 \\
\hline 5 & $7: 45$ & 30 & 64 & 2 \\
\hline 6 & $8: 15$ & 15 & 69 & 2 \\
\hline 7 & $7: 15$ & 15 & 53 & 2 \\
\hline 8 & $7: 30$ & 30 & 57 & 3 \\
\hline 9 & $8: 00$ & 15 & 65 & 2 \\
\hline 10 & $8: 15$ & 15 & 65 & 3 \\
\hline 11 & $8: 45$ & 15 & 58 & 3 \\
\hline 12 & $7: 30$ & 30 & 48 & 1 \\
\hline 13 & $8: 00$ & 30 & 47 & 1 \\
\hline 14 & $8: 45$ & 30 & 46 & 1 \\
\hline 15 & $8: 00$ & 15 & 52 & 1 \\
\hline 16 & $8: 15$ & 30 & 62 & 2 \\
\hline 17 & $9: 00$ & 15 & 58 & 1 \\
\hline 18 & $9: 15$ & 30 & 47 & 2 \\
\hline 19 & $8: 00$ & 30 & 48 & 3 \\
\hline 20 & $8: 30$ & 30 & 45 & 3 \\
\hline 21 & $9: 15$ & 15 & 55 & 2 \\
\hline 22 & $9: 30$ & 30 & 56 & 3 \\
\hline
\end{tabular}

Table 5 Input file 2 testcase 3

\begin{tabular}{|c|c|c|c|c|c|c|}
\hline ID & $\begin{array}{l}\text { Name care } \\
\text { worker }\end{array}$ & $\begin{array}{l}\text { Qualifica- } \\
\text { tion Level }\end{array}$ & $\begin{array}{l}\text { Start time } \\
\text { shift }\end{array}$ & $\begin{array}{l}\text { End time } \\
\text { shift }\end{array}$ & $\begin{array}{l}\text { Prefered } \\
\text { break time }\end{array}$ & $\begin{array}{l}\text { Break } \\
\text { duration }\end{array}$ \\
\hline 1 & Olivia & & $7: 00$ & $8: 15$ & $7: 45$ & 15 \\
\hline 2 & Arthur & 2 & $7: 00$ & $8: 30$ & $7: 30$ & 15 \\
\hline 3 & Theo & 3 & $7: 15$ & $9: 00$ & $8: 30$ & 15 \\
\hline 4 & Emily & 1 & $7: 30$ & $9: 15$ & $8: 30$ & 15 \\
\hline 5 & Sophia & 2 & $8: 00$ & $9: 45$ & $8: 45$ & 15 \\
\hline 6 & Clara & 3 & $8: 00$ & 10:00 & 9:00 & 15 \\
\hline
\end{tabular}

